\title{
The Impact of Tube Optimization Design on Radiator Heat Rejection Performance
}

\author{
Toure Ismael and Buyun Sheng \\ Department of Mechanical and Electronic Engineering, Wuhan University of Technology, Wuhan, China (NIGER)
}

\begin{abstract}
This work consists to evaluate the impact of the tube design on the car radiator heat rejection performance. For this work we come up with two propositions, the first one consists to change the traditional tube shape "1.6x16 B-type" to "1.6x16 Dot B- type". The second one is just to change the traditional tube dimension from $1.6 \times 16$ to $1.4 \times 16$. After the two samples radiators were made, we will proceed to their performance analysis such as the heat rejection, the coolant pressure drops, and the airside pressure drop. For these performance tests we will use the company laboratory Multi-functional test bench (T-HWS-2H), and some mathematical formula to calculate our samples performance. At last we will compare our analysis results with the radiator "1.6x16 B-type" heat rejection data (Req.), which structural model and data is our research basic standard. The results show that the tube design has great impact on the radiator heat rejection performance.

This work has been performed at HUBEI RADIATECH COOLING SYSTEM CO., LDT (HBR).
\end{abstract}

Keywords- car radiator; tube type; fin

\section{INTRODUCTION}

The air-cooled heat exchangers found in a vehicle (radiator, AC condenser and evaporator, charge air cooler, etc.) has an important role in its weight and also in the design of its frontend module, which also has a strong impact on the car aerodynamic behavior. The radiator is the main component in a cooling system to remove heat from the engines. Looking at these challenges, an optimization process is compulsory to obtain the best design compromise between performance, size/ shape and weight. This experience's objective demands advanced design tools that can indicate not only the best solution but also the fundamental reason of a performance improvement that will satisfy our customers and market demand. To improve the heat transfer from the surface, we use the $\mathrm{B}$ shape tubes which are vital components in the radiator design, the liquid flows in B shape tubes while the air flows in channels set up by multileveled fin surfaces. In many situations, the thermal resistance on the air side is larger than that on the liquid side. Lu et al. [1-2] also adopted the porous medium model to simulate the pressure loss through the horizontal radiator in a small dry cooling tower. [3-4] analyzed the performance of a spiral tube heat exchanger, in comparison with shell and tube heat exchanger. Their optimized spiral design revealed that, heat transfer is enhanced compared to the shell and tube heat exchanger. Different experimental studies were performed to analyze and verify their advantages in various heat exchange systems like shell and tube heat exchangers [5], double tube heat exchangers [6-7], plate heat exchangers [8], heat pipes [9-10], microchannel heat sink [11], electronics cooling [12], building air conditioning [13], and the like. Leong et al. [14] attempted to investigate the heat transfer characteristics of an automotive car radiator using ethylene glycol based copper nanofluids numerically Ismael,T et al [15] presented a set of parametric studies of heat dissipation performed on automotive radiators by of designing five radiators with different fin pitch wave distance $(\mathrm{P}=2.5$, 2.4, 2.3, 2.2, $2.1 \mathrm{~mm})$.

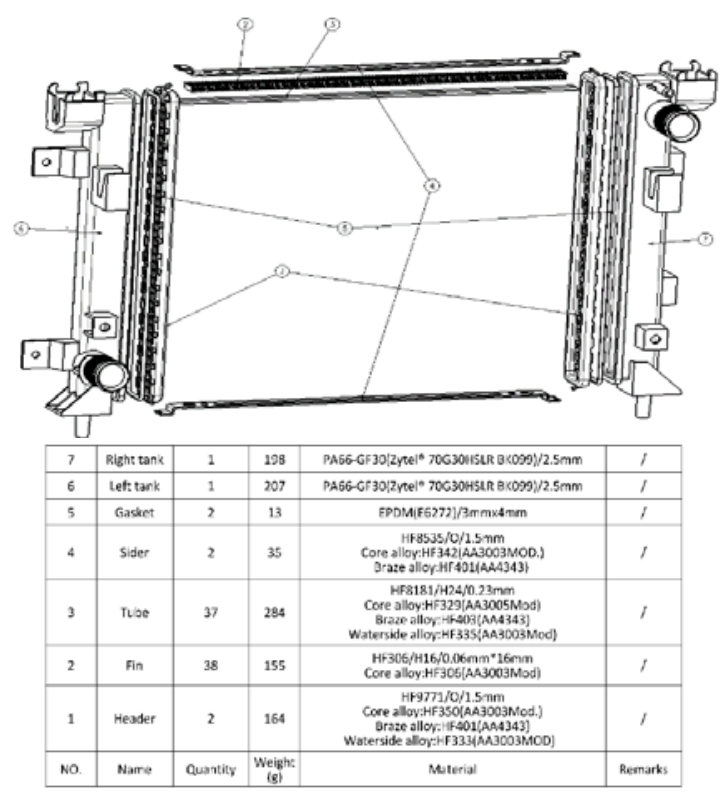

FIGURE I.RADIATOR STRUCTURAL DESIGN AND NOMENCLATURE

\section{II.EXPERIENCE PROCEDURE AND RESULTS}

The analysis focuses on the cooling performance for automobile radiator by changing the tube design and parameters. For the cooling performance experience, we use T-HWS-2H Multi-functional test bench for Automobile and Tractor Radiators. The test bench system is a continuous air suction type wind tunnel in a chamber which can also control the ambient air temperature; collection and control of operating condition parameters can be done automatically by the computer via the preset program, and also can be done by the user manually. We also use some mathematical formula according to the heat dispassion factors we develop in the company. 


\section{A. First Proposition}

We use CATIA V5-6R2014 to draw our designed sample, which consist to change the tube type from "1.6x16 B-type" to "1.6x16 B-Dot type" as shown in figure 2. After the new sample is made we precede to his analysis true the wind tunnel in the company laboratory.
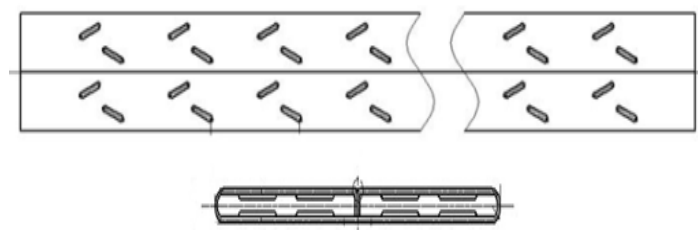

1. $6 \times 16$ Dot type
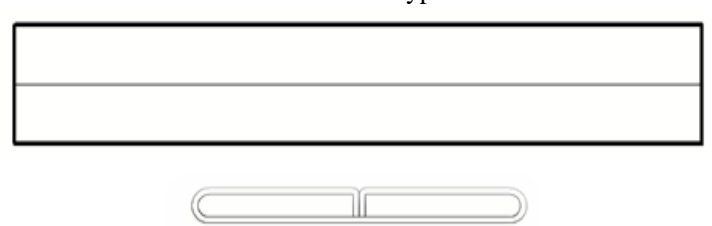

1.6x16 B-type

FIGURE II.

TUBE STRUCTURAL DESIGNS

\section{ANALYSIS RESULTS}

TABLE I. WIND TUNNEL BENCH ANALYSIS RESULTS DATA

\begin{tabular}{|c|c|c|c|c|c|c|c|c|}
\hline \multicolumn{2}{|c|}{ Pth (KW) } & \multicolumn{7}{|c|}{$T$ coolant $=90^{\circ} \mathrm{C}$} \\
\hline & & 120 & 240 & 360 & 480 & 720 & 840 & Pext \\
\hline \multirow{7}{*}{$\begin{array}{l}\text { Tamb }= \\
20^{\circ} \mathrm{C}\end{array}$} & $1 \mathrm{~m} / \mathrm{s}$ & 7.4 & 7.7 & 7.8 & 7.8 & 7.8 & 7.8 & 6.5 \\
\hline & $2 \mathrm{~m} / \mathrm{s}$ & 12.5 & 14. & 14.7 & 15.1 & 15.5 & 15.5 & 25.7 \\
\hline & $\begin{array}{c}3.5 \mathrm{~m} \\
/ \mathrm{s}\end{array}$ & 16.9 & $\begin{array}{c}20 . \\
5\end{array}$ & 22.7 & 24.2 & 25.9 & 26.3 & 68.1 \\
\hline & $5 \mathrm{~m} / \mathrm{s}$ & 20.0 & $\begin{array}{c}24 . \\
5\end{array}$ & 27.3 & 29.5 & 32.8 & 34.0 & 107.2 \\
\hline & $\begin{array}{c}6.5 \mathrm{~m} \\
/ \mathrm{s}\end{array}$ & 22.1 & $\begin{array}{c}27 . \\
1\end{array}$ & 29.4 & 31.2 & 34.3 & 35.6 & 157.6 \\
\hline & $8 \mathrm{~m} / \mathrm{s}$ & 23.5 & $\begin{array}{c}29 . \\
9\end{array}$ & 33.5 & 36.6 & 41.7 & 43.9 & 209.4 \\
\hline & $\begin{array}{c}\triangle \\
\text { Pint } \\
\text { mba } \\
\text { r) } \\
@ 5 \\
\text { m/s }\end{array}$ & 40.0 & $\begin{array}{c}154 \\
.5\end{array}$ & $\begin{array}{c}348 . \\
0\end{array}$ & $\begin{array}{c}618 . \\
9\end{array}$ & $\begin{array}{l}139 \\
3.2\end{array}$ & $\begin{array}{l}189 \\
6.7\end{array}$ & \\
\hline
\end{tabular}

TABLE II. REQ AND HBR DATA COMPARISON

\begin{tabular}{|c|c|c|c|c|c|c|c|}
\hline $\begin{array}{c}\text { Operating } \\
\text { point }\end{array}$ & $\begin{array}{c}\text { Air } \\
\text { speed } \\
{[\mathbf{m} / \mathbf{s}]}\end{array}$ & $\begin{array}{c}\text { Tamb } \\
{\left[^{\circ} \mathbf{C}\right]}\end{array}$ & $\begin{array}{c}\text { Coolant } \\
\text { Flow } \\
{[\mathbf{L} / \mathbf{m i n}]}\end{array}$ & $\begin{array}{c}\mathbf{T} \\
\text { inlet } \\
{\left[^{\circ}\right.} \\
\mathbf{C}]\end{array}$ & $\begin{array}{c}\text { Heat } \\
\text { Exchange } \\
{[\mathrm{Kw}]}\end{array}$ & $\begin{array}{c}\triangle \text { Pext } \\
\text { Max } \\
{[\mathbf{P a}]}\end{array}$ & $\begin{array}{c}\triangle \\
\text { Pint } \\
\text { Max } \\
{[\mathrm{Kpa}]}\end{array}$ \\
\hline Req. & \multirow{2}{*}{$\mathbf{2 . 0 0}$} & $\mathbf{2 0}$ & $\mathbf{1 6}$ & $\mathbf{9 0}$ & $\mathbf{1 1 . 2 0}$ & $\mathbf{2 8 . 2 0}$ & 10.85 \\
\hline HBR & & $\mathbf{2 0}$ & $\mathbf{1 6}$ & $\mathbf{9 0}$ & $\mathbf{1 1 . 7 0}$ & $\mathbf{2 5 . 6 0}$ & 2.62 \\
\hline Req. & \multirow{2}{*}{5.00} & $\mathbf{2 0}$ & $\mathbf{1 6}$ & $\mathbf{9 0}$ & $\mathbf{1 7 . 0 0}$ & $\mathbf{1 1 6 . 6 0}$ & 10.85 \\
\cline { 3 - 8 } HBR & $\mathbf{2 0}$ & $\mathbf{1 6}$ & $\mathbf{9 0}$ & $\mathbf{1 7 . 9 0}$ & $\mathbf{1 0 6 . 3 0}$ & 2.68 \\
\hline
\end{tabular}

- The impact of B-Dot type shape tube on the heat rejection parameters
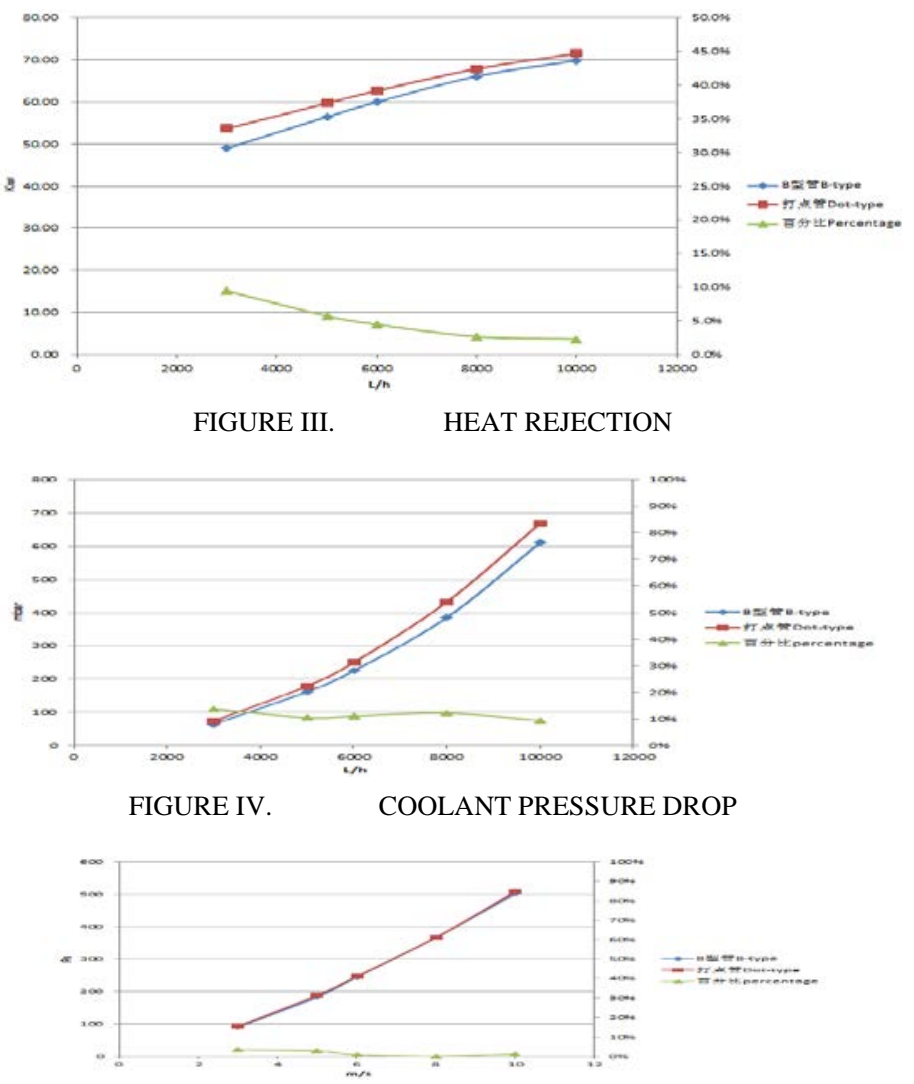

FIGURE V. AIRSIDE PRESSURE DROP

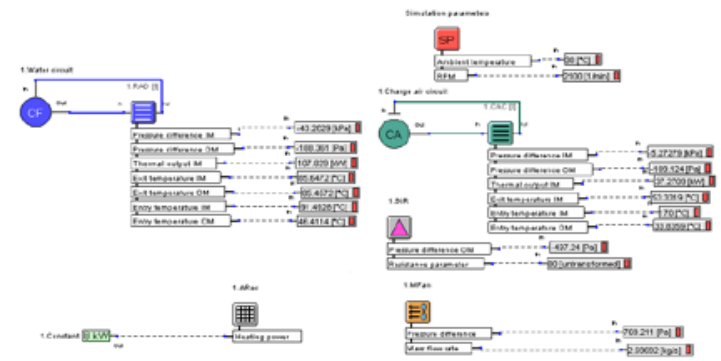

FIGURE VI.

KULI PERFORMANCE SIMULATION

According to our research and analysis results, after we changed the tube B-shape to Dot B-type, it has great impact on the radiator working condition. From fig 3 to fig 5 we can observe that the radiator heat rejection increases about $5 \%$, coolant pressure drops about $15 \%$, and the airside pressure drop remain almost invariant. As we know from the radiator working condition the smaller the flow rate is, the higher the heat rejection will increase. That's means the Dot B-shape tube reaches our goal which is to increase the radiator heat dissipation performance, but it's also improve the tube structural strength. From our analysis experience data on the radiator and other products we ve work on in the past, we know that if the heat rejection decreases by $1 \%$, airside pressure drop will decrease by $3 \%$. According to this relation between the two factors, if we decrease the heat rejection by $5 \%$, the airside pressure drop will also decrease by $15 \%$, as shown in the performance data from tab 3 to tab 4 . After decreasing the heat rejection by $5 \%$, the sample we design meet the requirements, but still have one point who is still 
higher (yellow part in tab 4) than the standard sample data (Req.). But that is not a problem because the radiator we use in this work has a small flow rate, that's means the heat rejection improvement may exceed $5 \%$. So, there will be more space to adjust the airside pressure drop. In the future we will make some new samples to measure the performance value, and then make some adjustment.

TABLE III. REQ AND HBR DATA COMPARISON (1.6X16 BTYPE )

\begin{tabular}{|c|c|c|c|c|c|c|c|}
\hline \multirow{3}{*}{\multicolumn{2}{|c|}{$\begin{array}{l}\text { Radiator Heat Rejection } \\
\text { (KW) }\end{array}$}} & \multicolumn{4}{|c|}{ Air Velocity $\mathrm{m} / \mathrm{sec}(\mathrm{rt}=70 \mathrm{deg})$} & \multirow{2}{*}{\multicolumn{2}{|c|}{$\begin{array}{c}\text { Coolant } \\
\text { Pressure Drop } \\
\text { (Kpa) } \\
\end{array}$}} \\
\hline & & \multicolumn{2}{|c|}{$2 \mathrm{~m} / \mathrm{s}$} & \multicolumn{2}{|c|}{$5 \mathrm{~m} / \mathrm{s}$} & & \\
\hline & & Req. & HBR & Req. & HBR & Req. & HBR \\
\hline \multirow{2}{*}{$\begin{array}{c}\text { Coolant } \\
\text { Flow }\end{array}$} & 16L/Min & 10.5 & 11.0 & & & 10.85 & 2.85 \\
\hline & 16L/Min & & & 14.7 & 17.0 & 10.85 & 2.85 \\
\hline \multicolumn{2}{|c|}{ Airside Pressure Drop (pa) } & 26 & 36.3 & 110.0 & 126.7 & & \\
\hline
\end{tabular}

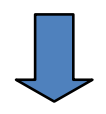

TABLE IV.
Heat rejection $5 \%$

Airside pressure drop 15\%

REQ AND HBR DATA COMPARISON (1.6X16 DOT TYPE)

\begin{tabular}{|c|c|c|c|c|c|c|c|}
\hline \multirow{3}{*}{\multicolumn{2}{|c|}{$\begin{array}{c}\text { Radiator Heat Rejection } \\
\text { (KW) }\end{array}$}} & \multicolumn{4}{|c|}{ Air Velocity m/sec (rt =70 deg) } & \multirow{2}{*}{\multicolumn{2}{|c|}{$\begin{array}{c}\text { Coolant } \\
\text { Pressure Drop } \\
\text { (Kpa) }\end{array}$}} \\
\hline & & \multicolumn{2}{|c|}{$2 \mathrm{~m} / \mathrm{s}$} & \multicolumn{2}{|c|}{$5 \mathrm{~m} / \mathrm{s}$} & & \\
\hline & & Req. & HBR & Req. & HBR & Req. & HBR \\
\hline \multirow{2}{*}{$\begin{array}{l}\text { Coolant } \\
\text { Flow }\end{array}$} & 16L/Min & 10.5 & 10.5 & & & 10.85 & 2.85 \\
\hline & 16L/Min & & & 14.7 & 16.3 & 10.85 & 2.85 \\
\hline \multicolumn{2}{|c|}{ Airside Pressure Drop (pa) } & 26 & 30.9 & 110.0 & 107.7 & & \\
\hline
\end{tabular}

\section{B. Second Proposition}

This solution consists to change our standard prototype tube size from $1.6 \times 16$ to $1.4 \times 16$, and then run some heat rejection performance analysis true mathematical formula and the laboratory heat rejection test bench.
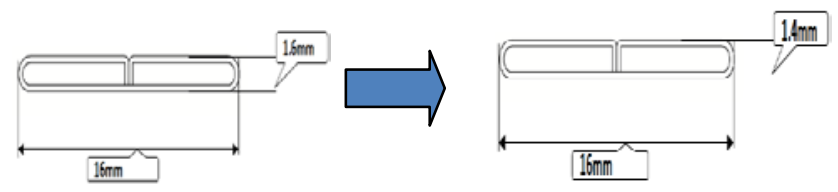

FIGURE VII. B SHAPE TUBE FROM $1.6 \times 16$ to $1.4 \times 16$

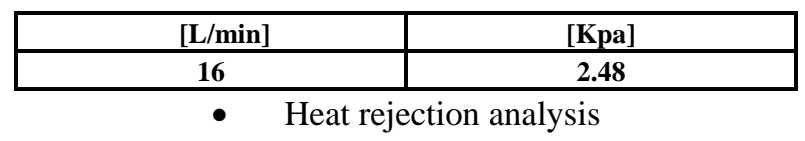

$\mathrm{Pth}=\mathrm{K} * \mathrm{~A} * \Delta \mathrm{T}$

Pth - Heat rejection

K —Transfer coefficient

A —Surface area

$\Delta \mathrm{T}$-Temperature difference

th $=1 /(1 / \mathrm{h} 1 / \mathrm{A} 1+\delta / \lambda / \mathrm{A} 1+1 / \mathrm{h} 2 / \mathrm{A} 2) *(\mathrm{t} 1-\mathrm{t} 4)$

h1-Internal Convection coefficient

h2_External Convection coefficient

A1_-Internal surface area

A2__External surface area

$\lambda-$ Heat conductivity coefficient

$\delta$-Tube thickness

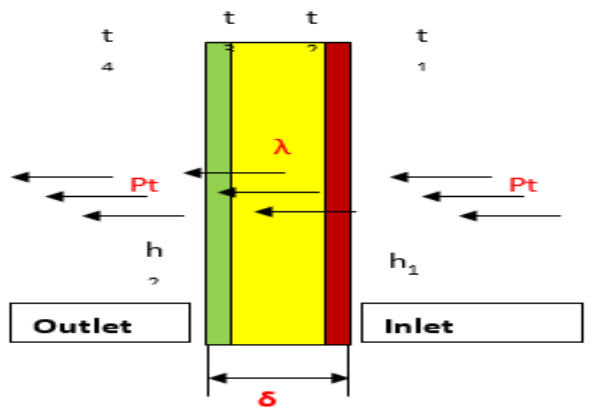

There are three principal parameters, $\mathrm{K}, \mathrm{A}$ and $\Delta \mathrm{T}$ that affect the radiator heat rejection. In general, $\Delta \mathrm{T}$ is almost invariant, so we only need to analyze $\mathrm{K}$ and A parameters.

When the core size is constant, the tube height changed from 1.6 to 1.4 , and then the ventilation area is increased from 0.741 to 0.7605 . The airside pressure drop can absolutely be decreased; also, the $\mathrm{A}$ and $\mathrm{K}$ value can be increased respectively by $2.5 \%, 3 \%$. That`s confirm again our sample perfectly reaches the requirements.

- Coolant Pressure Drop analysis

In the case the tank structure is constant $\Delta \mathbf{P i n t}=\mathbf{k V}^{\mathbf{2}}$.

$\Delta$ Pint - Coolant Pressure Drop

V_Flow velocity per tube
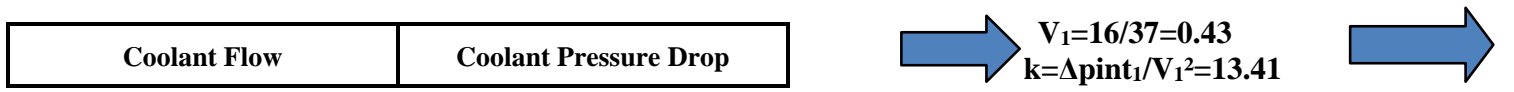

$V_{2}=16 / 38=0.42$

$\Delta$ pint $_{2}=\mathbf{k V}_{2}{ }^{2}=2.37$

TABLE V.HBR SIMULATION DATA

\begin{tabular}{|c|c|c|c|c|c|c|c|c|c|}
\hline $\begin{array}{c}\text { Operating } \\
\text { point }\end{array}$ & $\begin{array}{c}\text { Engine } \\
\text { rmp }\end{array}$ & $\begin{array}{c}\text { Vehicle } \\
\text { speed }\end{array}$ & $\begin{array}{c}\text { Air } \\
\text { speed }\end{array}$ & $\mathbf{T}^{\circ} \mathbf{a m b}$ & $\begin{array}{c}\text { Q } \\
\text { liquid }\end{array}$ & $\begin{array}{c}\mathrm{T}^{\circ} \\
\text { coolant } \\
\text { inlet }\end{array}$ & $\mathrm{A} / \mathrm{C}$ & $\begin{array}{c}\text { Pth } \\
\text { mini } \\
\text { target }\end{array}$ & $\begin{array}{l}\text { Pth } \\
\text { HBR }\end{array}$ \\
\hline & [tr/min] & [km/h] & {$[\mathrm{m} / \mathrm{s}]$} & {$\left[{ }^{\circ} \mathrm{C}\right]$} & [L/h] & {$\left[{ }^{\circ} \mathrm{C}\right]$} & [kW] & [kW] & [kW] \\
\hline Point 1 & 2845 & 50 & 3.07 & 32 & 3646 & 118 & 0 & 37.7 & 46.6 \\
\hline Point 2 & 3003 & 77 & 3.52 & 37 & 3849 & 118 & $\mathbf{0}$ & 38.5 & 48.3 \\
\hline Point 3 & 3432 & 88 & 3.71 & 45 & 4398 & 115 & 9 & 43.4 & 43.7 \\
\hline Point 4 & 3591 & 110 & 4.17 & 45 & 4602 & 115 & 9 & 45.2 & 47.9 \\
\hline Point 5 & 3510 & 90 & 3.74 & 32 & 4498 & 118 & $\mathbf{0}$ & 46.3 & 55.7 \\
\hline Point 6 & 4244 & 130 & 4.61 & 37 & 5439 & 110 & 7.5 & 55.7 & 55.8 \\
\hline Point 7 & 5306 & 200 & 5.89 & 45 & 6800 & 118 & $\mathbf{0}$ & 75.3 & 68.8 \\
\hline Point 8 & 5306 & 200 & 5.89 & 32 & 6800 & 118 & $\mathbf{0}$ & 76.6 & 82.5 \\
\hline Point 9 & 5466 & 206 & 5.97 & 25 & 7005 & 118 & $\mathbf{0}$ & 78.2 & 91.4 \\
\hline
\end{tabular}


TABLE VI DATA COMPARISON

\begin{tabular}{|c|c|c|c|c|c|c|c|c|c|}
\hline \multirow{2}{*}{ Point } & $\begin{array}{c}\text { Engin } \\
\mathrm{e} \\
\mathrm{rm}\end{array}$ & $\begin{array}{c}\text { Vehicle } \\
\text { speed }\end{array}$ & Air speed & $\mathrm{T}^{\circ}$ amb & Q liquid & $\begin{array}{c}\mathrm{T}^{\circ} \text { coolan } \\
\mathrm{t} \\
\mathrm{in}\end{array}$ & $\begin{array}{c}\text { Pth mini } \\
\text { target }\end{array}$ & $\begin{array}{c}\text { A/C } \\
\text { Pth HBR }\end{array}$ & $\mathrm{k}$ \\
\hline Point 1 & 2845 & 50 & 3.04 & 32 & 3720.8 & 118 & 0 & 37.7 & 49.1 \\
\hline Point 2 & 3003 & 77 & 3.49 & 37 & 3927.4 & 118 & 0 & 38.5 & 50.8 \\
\hline Point 3 & 3432 & 88 & 3.67 & 45 & 4488.5 & 115 & 9 & 43.4 & 45.7 \\
\hline Point 4 & 3591 & 110 & 4.12 & 45 & 4696.4 & 115 & 9 & 45.2 & 49.7 \\
\hline Point 5 & 3510 & 90 & 3.70 & 32 & 4590.5 & 118 & 0 & 46.3 & 58.0 \\
\hline Point 6 & 4244 & 130 & 4.55 & 37 & 5550.4 & 110 & 7.5 & 55.7 & 57.2 \\
\hline Point 7 & 5306 & 200 & 5.78 & 45 & 6939.4 & 118 & 0 & 75.3 & 69.1 \\
\hline Point 8 & 5306 & 200 & 5.78 & 32 & 6939.4 & 118 & 0 & 76.6 & 82.7 \\
\hline Point 9 & 5466 & 206 & 5.86 & 25 & 7148.6 & 118 & 0 & 78.2 & 91.5 \\
\hline
\end{tabular}

\begin{tabular}{|c|c|c|c|c|c|}
\hline Q liquid & $\begin{array}{c}\text { T liquid } \\
\text { at inlet }\end{array}$ & $\begin{array}{c}\text { Vair on } \\
\text { core }\end{array}$ & Tamb & $\begin{array}{c}\Delta \text { Pint max } \\
\text { GOAL }\end{array}$ & $\begin{array}{c}\Delta \text { Pint } \\
\text { HBR }\end{array}$ \\
\hline$[\mathrm{L} / \mathrm{h}]$ & {$\left[{ }^{\circ} \mathrm{C}\right]$} & {$[\mathrm{m} / \mathrm{s}]$} & {$\left[{ }^{\circ} \mathrm{C}\right]$} & {$[$ mbar $]$} & {$[\mathrm{mbar}]$} \\
\hline 3000 & 95 & 5 & 20 & & 99 \\
\hline 5000 & 95 & 5 & 20 & & 213 \\
\hline 6000 & 95 & 5 & 20 & 300 & 293 \\
\hline 8000 & 95 & 5 & 20 & & 506 \\
\hline 10000 & 95 & 5 & 20 & & 796 \\
\hline
\end{tabular}

\begin{tabular}{|c|c|c|c|c|c|}
\hline $\begin{array}{c}\mathrm{Q} \\
\text { liquid }\end{array}$ & $\begin{array}{c}\text { T liquid } \\
\text { at inlet }\end{array}$ & $\begin{array}{c}\text { V air } \\
\text { on core }\end{array}$ & Tamb & $\begin{array}{c}\Delta \text { Pext max } \\
\text { GOAL }\end{array}$ & $\begin{array}{c}\Delta \text { Pext } \\
\text { HBR }\end{array}$ \\
\hline$[\mathrm{L} / \mathrm{h}]$ & {$\left[{ }^{\circ} \mathrm{C}\right]$} & {$[\mathrm{m} / \mathrm{s}]$} & {$\left[{ }^{\circ} \mathrm{C}\right]$} & {$[\mathrm{mbar}]$} & {$[\mathrm{mbar}]$} \\
\hline 6000 & 95 & 3 & 20 & & 94 \\
\hline 6000 & 95 & 5 & 20 & & 196 \\
\hline 6000 & 95 & 6 & 20 & 200 & 254 \\
\hline 6000 & 95 & 8 & 20 & & 385 \\
\hline 60000 & 95 & 10 & 20 & & 556 \\
\hline
\end{tabular}

TABLE VII.

HBR TEST DATA

\begin{tabular}{|c|c|c|c|c|c|c|c|c|c|}
\hline $\begin{array}{c}\text { Operating } \\
\text { point }\end{array}$ & $\begin{array}{c}\text { Engine } \\
\text { rmp }\end{array}$ & $\begin{array}{c}\text { Vehicle } \\
\text { speed }\end{array}$ & $\begin{array}{c}\text { Air } \\
\text { speed }\end{array}$ & $\mathrm{T}^{\circ}$ amb & $\begin{array}{c}\mathrm{Q} \\
\text { liquid }\end{array}$ & $\begin{array}{c}\mathrm{T}^{\circ} \text { coolant } \\
\text { inlet }\end{array}$ & $\mathrm{A} / \mathrm{C}$ & $\begin{array}{c}\text { Pth } \\
\text { mini } \\
\text { target }\end{array}$ & $\begin{array}{c}\text { Pth } \\
\text { HBR }\end{array}$ \\
\hline Point 1 & 2845 & 50 & $\mathbf{3 . 0 4}$ & 32 & $\mathbf{3 7 2 1}$ & 118 & 0 & 37.7 & 49.1 \\
\hline Point 2 & 3003 & 77 & $\mathbf{3 . 4 9}$ & 37 & $\mathbf{3 9 2 7}$ & 118 & 0 & 38.5 & 50.8 \\
\hline Point 3 & 3432 & 88 & $\mathbf{3 . 6 7}$ & 45 & $\mathbf{4 4 8 9}$ & 115 & 9 & 43.4 & 45.7 \\
\hline Point 4 & 3591 & 110 & $\mathbf{4 . 1 2}$ & 45 & $\mathbf{4 6 9 6}$ & 115 & 9 & 45.2 & 49.7 \\
\hline Point 5 & 3510 & 90 & $\mathbf{3 . 7 0}$ & 32 & $\mathbf{4 5 9 1}$ & 118 & 0 & 46.3 & 58 \\
\hline Point 6 & 4244 & 130 & $\mathbf{4 . 5 5}$ & 37 & $\mathbf{5 5 5 0}$ & 110 & 7.5 & 55.7 & 57.2 \\
\hline Point 7 & 5306 & 200 & $\mathbf{5 . 7 8}$ & 45 & $\mathbf{6 9 3 9}$ & 118 & 0 & 75.3 & 69.1 \\
\hline Point 8 & 5306 & 200 & $\mathbf{5 . 7 8}$ & 32 & $\mathbf{6 9 3 9}$ & 118 & 0 & 76.6 & 82.7 \\
\hline Point 9 & 5466 & 206 & $\mathbf{5 . 8 6}$ & 25 & $\mathbf{7 1 4 9}$ & 118 & 0 & 78.2 & 91.5 \\
\hline
\end{tabular}

show that the modifications we made on the tube have

$\Delta$ Pint is representable when air speed is at $5 \mathrm{~m} / \mathrm{s}$, and $\Delta \mathrm{P}$ ext

is representable when coolant flow is at $6000 \mathrm{~L} / \mathrm{h}$. To evaluate our sample heat rejection, we will perform the same simulation with KULI software and analysis with the Multifunctional test bench as our standard prototype. The analysis consists to perform work test bench and simulation analysis of our radiator sample for nine different vehicle speeds (point 1 to 9). From our experience results as shown in tab 5-6-7 we can observe except the point 7 that our second prototype can meet the standard sample heat rejection requirements ( $\Delta$ Pint and $\Delta$ Pext).

\section{CONCLUSION}

In this paper, we change the radiator tube design to improve the heat dissipation performance. Our analysis results significant impact on the radiator heat rejection, and also increase his structure strength. But we still have to further our study on the radiator working performance (heat rejection, structure fatigue durability, strength ect ), because the car industry have more and more requirements on the engine working, and the radiator is the most important part on the cooling system.

\section{ACKNOWLEDGEMENTS}

The first author would like to express his gratitude to Hubei Radiatech Auto Cooling System Co., LTD for giving us the chance to use the company lab to perform our experience, to our supervisor Mr. Sheng Bu Yun, to Mr. Shi Rui and Mr.Li Xing Yuan for their prolonged research collaboration and for providing valuable information 


\section{REFERENCES}

[1] Lu, Y., Guan, Z., Gurgenci, H. and Zou, Z. (2013) Windbreak Walls Reverse the Negative Effect of Crosswind in Short Natural Draft Dry Cooling Towers into a Performance Enhancement. International Journal of Heat and Mass Transfer; 63, 162-170.

[2] Lu, Y., Gurgenci, H., Guan, Z. and He, S. (2014) The Influence of Windbreak Wall Orientation on the Cooling Performance of Small Natural Draft Dry Cooling Towers. International Journal of Heat and Mass Transfer, 79, 1059-1069.

[3] Akhilnandh Ramesh*, M. Jaya Arun Prasanth, A.Kirthivasan, M.Suresh, Heat Transfer Studies on Air Cooled Spiral Radiator with Circumferential Fins, International Conference on Computational Heat and Mass Transfer-127( 2015 ) 333 - 339 , doi: 10.1016/j.proeng.2015.11.378

[4] M. Bidabadi, A.K.Sadaghiani, A.Vahdat Azad., Spiral Heat Exchanger Optimization using Genetic Algorithm, ScientiaIranica B: Transactions in Mechanical Engineering, 20(5), (2013), pp.1445-1454.

[5] B. Farajollahi, S.Gh. Etemad, M. Hojjat, Heat transfer of nanofluids in a shell and tube heat exchanger, Int. J. Heat Mass Transfer 53 (2010) 12e17.

[6] A. Zamzamian, S.N. Oskouie, A. Doosthoseini, A. Joneidi, M. Pazouki, Experimental investigation of forced convective heat transfer coefficient in nanofluids of Al2O3/EG and CuO/EG in a double pipe and plate heat exchangers under turbulent flow, Exp. Therm. Fluid Sci. 35 (3) (2011).

[7] K.Y. Leong, R. Saidur, S.N. Kazi, A.H. Mamun, Performance investigation of an automotive car radiator operated with nanofluidbased coolants (nanofluid as a coolant in a radiator), Appl. Therm. Eng. 30 (2010).

[8] R.S. Vajjha, D.K. Das, P.K. Namburu, Numerical study of fluid dynamic and heat transfer performance of $\mathrm{Al} 2 \mathrm{O} 3$ and $\mathrm{CuO}$ nanofluids in the flat tubes of a radiator, Int. J. Heat Fluid Flow 31 (4) (2010) 613e621.

[9] C. Cuevas, D. Makaire, L. Dardenne, P. Ngendakumana, Thermohydraulic characterization of a louvered fin and flat tube heat exchanger, Exp. Therm. Fluid Sci. 35 (2011) 154e164.

[10] A.A. Avramenko, D.G. Blinov, I.V. Shevchuk, Self-similar analysis of fluid flow and heat-mass transfer of nanofluids in boundary layer, Phys. Fluids 23 (2011) 082002.

[11] S.M. Peyghambarzadeh, S.H. Hashemabadi, M.S. Jamnani, S.H. Hoseini, Improving the cooling performance of automobile radiator with Al2O3/water nanofluid, Appl. Therm. Eng. 31 (2011) 1833e1838.

[12] S.M. Peyghambarzadeh, S.H. Hashemabadi, S.H. Hoseini, M.S. Jamnani, Experimental study of heat transfer enhancement using water/ethylene glycol based nanofluids as a new coolant in the car radiator, Int. Commun. Heat Mass Transfer 38 (2011) 1283e1290.

[13] L. Fedele, L. Colla, S. Bobbo, S. Barison, F. Agresti, Experimental stability analysis of different waterbased nanofluids, Nanoscale Res. Lett. 6 (2011) 300e307.

[14] C.S. Jwo, L.Y. Jeng, T.P. Teng, C.C. Chen, Performance of overall heat transfer in multi-channel heat exchanger by alumina nanofluid, J. Alloys Compd 504S (2010) S385eS388.

[15] Ismael, T., Yun, S.B. and Ulugbek, F. (2016) Radiator Heat Dissipation Performance. Journal of Electronics Cooling and Thermal Control, 6, 8896. 